\title{
CELLS AND CELLULARITY IN INFINITE-DIMENSIONAL NORMED LINEAR SPACES
}

BY

\author{
R. A. MC $\operatorname{COY}(1)$
}

ABSTRACT. Certain concepts such as cells, cellular sets, point-like sets, and decomposition spaces are studied and related in normed linear spaces. The relationships between these concepts in general resemble somewhat the corresponding relationships in Euclidean space.

There are certain topological properties in Euclidean $n$-space which can be conveniently studied as properties in normed linear spaces. In this paper concepts such as open and closed cells, cellular sets, point-like sets, and decomposition spaces are studied and related. Many, but not all, of the relationships between these concepts in infinite-dimensional normed linear spaces resemble the corresponding relationships in finite-dimensional spaces.

Throughout the paper, $E$ will denote an arbitrary normed linear space, and $\theta$ will represent the zero element of $E$. For any positive real number $r$ and any $x \in E$, let $B_{r}(x)=\{y \in E:\|x-y\| \leq r\}$ and $S_{r}(x)=\{y \in E:\|x-y\|=r\}$. For convenience let $B_{r}=B_{r}(\theta)$ and $S_{r}=S_{r}(\theta)$.

1. Tame cells. A closed subset $C$ of $E$ is a cell in $E$ if there exists a homeomorphism from the pair $\left(B_{1}, S_{1}\right)$ onto the pair $(C, \mathrm{Bd} C) . C$ is tame if there exists a homeomorphism from $E$ onto itself taking $C$ onto $B_{1}$. A closed subset $K$ of $E \backslash$ Int $C$ is a collar of $C$ if there exists a homeomorphism $b$ from the triple $\left(B_{1} ; B_{1 / 2}, S_{1}\right)$ onto the triple $(K \cup C ; C, B d(K \cup C))$.

Lemma 1.1. Let $C$ be a cell in $E$, and let $f$ be a bomeomorphism from the pair $\left(B_{1}, S_{1}\right)$ onto the pair $(C, \mathrm{Bd} C)$. Then there exists a bomeomorphism $b$ from $E$ onto itself such that $\left.b\right|_{B_{1 / 2}}=\left.f\right|_{B_{1 / 2}}$.

Presented to the Society, April 20,1968; received by the editors September 13, 1971 and, in revised form, February 23, 1972.

AMS (MOS) subject classifications (1970). Primary 57A17, 57A60; Secondary 57A20.

Key words and phrases. Normed linear spaces, open and closed cells, cellular sets, point-like sets, decomposition spaces, Hilbert space, annulus conjecture, monotone union theorem, shape of a point.

(1) This paper consists of a portion of the author's dissertation at lowa State University, written under D. E. Sanderson. The research was done in part while the author held a NASA fellowship and in part while he held an Iowa State Graduate Research Foundation fellowship. 
Proof. Set $D_{i}=B_{\left(2^{i}-1\right) / 2^{i}}$, for $i=1,2, \ldots$. Since there is a homeomorphism from $E$ onto itself which takes $C$ into $B_{1}$ and $f(\theta)=\theta$, it can be assumed without loss of generality that $C \subset B_{1}$ and $f(\theta)=\theta$. Choose $\epsilon>0$ so that $B_{\epsilon} \subset f\left(D_{1}\right)$, and choose $\delta>0$ so that $B_{\delta} \subset f^{-1}\left(B_{\epsilon / 2}\right)$. Define a homeomorphism $F_{1}$ from $E$ onto itse lf so that $F_{1}\left(D_{1}\right)=B_{\delta}$ and $\left.F_{1}\right|_{E \backslash D_{2}}=$ identity. Let $G_{1}$ be a homeomorphism from $E$ onto itself such that $G_{1}\left(B_{\epsilon}\right)=B_{2}$ and $\left.G_{1}\right|_{B_{\epsilon} / 2 \cup\left(E \backslash B_{3}\right)}=$ identity. Define the homeomorphism $f_{1}$ from $E$ onto itself by $f_{1}(x)=f F_{1} f^{-1}(x)$ if $x \in f\left(D_{2}\right)$, and $f_{1}(x)=x$ otherwise. Then define $b_{1}$, a homeomorphism from $E$ onto itself, by $b_{1}=f_{1}^{-1} G_{1} f_{1}$.

Suppose homeomorphisms $b_{i}$ from $E$ onto itself have been defined for $1 \leq$ $i \leq k$ such that $\left.b_{i}\right|_{b_{i-1} b_{i-2} \cdots b_{1} f\left(D_{i}\right)}=$ identity (for $i=1,\left.b_{i}\right|_{f\left(D_{i}\right)}=$ identity), $B_{2 i} \subset b_{i-1} b_{i-1} \cdots b_{1} f\left(D_{i+1}\right)$, and $b_{i} b_{i-1} \cdots b_{1} f\left(B_{1}\right) \subset B_{2 i+1}$. Then inductively define $b_{k+1}$ in the following manner. Let $F$ be a homeomorphism from $E$ onto itself such that $F\left(D_{k+1}\right)=D_{k}$ and $\left.F\right|_{E \backslash D_{k+2}}=$ identity, and let $G$ be a homeomorphism from $E$ onto itself such that $G\left(B_{2 k}\right)=B_{2 k+2}$ and $\left.G\right|_{B_{2 k-1} \cup\left(E \backslash B_{2 k+3}\right)}$ $=$ identity. Define the homeomorphism $\phi$ from $E$ onto itself by $\phi(x)=b_{k} \cdots$ $b_{1} f F f^{-1} b_{1}^{-1} \cdots b_{k}^{-1}(x)$ if $x \in b_{k} \cdots b_{1} f\left(D_{k+2}\right)$, and $\phi(x)=x$ otherwise. Then define $b_{k+1}$ by $b_{k+1}=\phi^{-1} G \phi$. Let $g$ be a homeomorphism from $E$ onto Int $B_{1}$ such that $\left.g\right|_{B_{1 / 2}}=$ identity. Then $b=\cdots b_{2} b_{1} f g$ is the desired homeomorphism.

The next theorem follows from Lemma 1.1 (also see [15]).

Theorem 1.1. A cell in $E$ is tame if and only if it has a collar.

Sanderson has given an example in [15] of a cell in Hilbert space which is not tame.

Lemma 1.2. Let $C$ and $C^{\prime}$ be two cells in $E$ such that $C^{\prime} \subset C$ and $C \backslash$ Int $C^{\prime}$ contains a collar $K$ of $C^{\prime}$. Then there exists a bomeomorphism $\phi$ from the pair $\left(B_{1}, S_{1}\right)$ onto the pair $(C, \mathrm{Bd} C)$ such that $C^{\prime} \subset \phi\left(B_{1 / 2}\right) \subset K \cup C^{\prime}$.

Proof. Let $g$ be a homeomorphism from $\left(B_{1}, S_{1}\right)$ onto $(C, \mathrm{Bd} C)$ and let $b$ be a homeomorphism from $\left(B_{1} ; B_{1 / 2}, S_{1}\right)$ onto $\left(K \cup C^{\prime} ; C^{\prime}, \operatorname{Bd}\left(K \cup C^{\prime}\right)\right)$. Choose $x \in B_{1}$ and $\epsilon>0$ so that $B_{\epsilon}(x) \subset g^{-1}$ (Int $\left.C^{\prime}\right)$. Let $f$ be a homeomorphism from $B_{1}$ onto itself so that $f\left(B_{1 / 2}\right)=B_{\epsilon}(x)$ and $\left.f\right|_{S_{1}}=$ identity. Choose $y \in B_{1}$ and $\delta>0$ so that $B_{\delta}(y) \subset b^{-1} g\left(B_{\epsilon}(x)\right)$. Let $F$ be a homeomorphism from $B_{1}$ onto itself so that $F\left(B_{\delta}(y)\right)=B_{1 / 2}$ and $\left.F\right|_{S_{1}}=$ identity. Define $G$, a homeomorphism from $C$ onto itself, by $G(x)=b F b^{-1}(x)$ if $x \in K \cup C^{\prime}$, and $G(x)=x$ otherwise. Then $G g f$ is the desired homeomorphism.

Lemma 1.3. Let $C$ be a cell in $E$ contained in $B_{1}$ such that $B_{1}$ contains a collar of $C$. Then there exists a bomeomorphism $b$ from Int $B_{1}$ onto itself such that $b\left(B_{1 / 2}\right)=C$. 
Proof. By Lemma 1.2, it can be assumed without loss of generality that $C \subset B_{1 / 2}$. If $K$ is a collar of $C$ contained in $B_{1} \backslash$ Int $C$, then there exists a homeomorphism $f$ from $\left(B_{1} ; B_{1 / 2}, S_{1}\right)$ onto $(K \cup C ; C, \mathrm{Bd}(K \cup C))$. By Lemma 1.1, there is a homeomorphism $F$ from $E$ onto itself such that $\left.F\right|_{B_{1 / 2}}=\left.f\right|_{B_{1 / 2}}$. Let $g$ be a homeomorphism from Int $B_{1}$ onto $E$ so that $\left.g\right|_{B \frac{1}{2}}=$ identity. Then define the desired homeomorphism $b$ to be $g^{-1} \mathrm{Fg}$.

Lemma 1.4. If $C$ and $D$ are closed subsets of $E$ contained in Int $B_{1}$ and $E \backslash B_{1}$ respectively, then there exist cells $C^{\prime}$ and $D^{\prime}$ in $E$ contained in Int $B_{1}$ and $E \backslash B_{1}$ respectively, such that $C \subset$ Int $C^{\prime}, D \subset E \backslash D^{\prime}, B_{1} \backslash$ Int $C^{\prime}$ is a collar of $C^{\prime}$, and $D^{\prime} \backslash$ Int $B_{1}$ is a collar of $B_{1}$.

Proof. To construct $C^{\prime}$ assume without loss of generality that $\theta \in C$. Let $d(x, C)=\inf \{\|x-z\|: z \in C\}$, and define the function $f$ from $S_{1 / 2}$ into Int $B_{1} \backslash C$ by $f(x)=[2-d(2 x, C)] x$. Since $d(\cdot, C)$ is a continuous function from $E$ onto the nonnegative real numbers, $f$ is continuous. Also since $f$ maps each point $x \in S_{1 / 2}$ into the line segment $[\theta: 2 x]$, then $f$ is one-to-one, and $f^{-1}$ is continuous. Define the homeomorphism $F$ from $B_{1}$ onto itself so that, for each $x \in S_{1 / 2}$, $F$ maps the line segment $[\theta: x]$ linearly onto $[\theta: f(x)]$ and maps the line segment $[x: 2 x]$ linearly onto $[f(x): 2 x]$. Then $F\left(B_{1 / 2}\right)$ is the desired cell $C^{\prime}$.

To construct $D^{\prime}$, define the function $g$ from $S_{1}$ into $E \backslash\left(D \cup B_{1}\right)$ by $g(x)=$ $[1+1 / 2 d(x, D)] x$. Define the homeomorphism $G$ from $B_{1}$ into $E \backslash D$ so that, for each $x \in S_{1}, G$ maps the line segment $[\theta: 1 / 2 x]$ linearly onto $[\theta: x]$ and maps the line segment $[1 / 2 x: x]$ linearly onto $[x: g(x)]$. Then $G\left(B_{1}\right)$ is the desired rell $D^{\prime}$.

The following "half-open annulus theorem" is well known for finite-dimensional spaces (see for example [12]).

Theorem 1.2. If $C$ is a tame cell in $E$ contained in Int $B_{1}$, then there exists a bomeomorphism $b$ from Int $B_{1}$ onto itself such that $h\left(B_{1 / 2}\right)=C$.

Proof. Let $f$ be a homeomorphism from $E$ onto itself such that $f(C)=B_{1}$. By Lemma 1.4, there exists a collar $K$ of $B_{1}$ so that $K \cup B_{1} \subset f\left(\right.$ Int $\left.B_{1}\right)$. Then $f^{-1}(K)$ is a collar of $C$ which is contained in Int $B_{1}$. The conclusion then follows from Lemma 1.3.

Corollary 1.1. Let $C$ and $C^{\prime}$ be two tame cells in $E$ such that $C^{\prime} \subset$ Int $C$. Then there exists a homeomorphism from the pair $\left(B_{1} \backslash B_{1 / 2}, S_{1}\right)$ onto the pair $\left(C \backslash C^{\prime}, \mathrm{Bd} C\right)$.

In general it is not known whether the half-open annulus Int $B_{1} \backslash$ Int $C$ in Theorem 1.2 can be strengthened to the annulus $B_{1} \backslash$ Int $C$. However, in [10] it is shown that the following conjecture is true for those normed linear spaces $E$ which are homeomorphic to a countable infinite product of copies of itself. 
Annulus conjecture for $E$. If $C$ is a tame cell in $E$ contained in Int $B_{1}$, then there exists a bomeomorphism $b$ from $B_{1}$ onto itself such that $b\left(B_{1 / 2}\right)=C$ and $\left.b\right|_{S_{1}}=$ identity.

If $C$ is a tame cell in $E$ contained in $B_{1 / 2}$, then $B_{1} \backslash$ Int $C$ is an approximation to an annulus in the following sense.

Theorem 1.3. Let $C$ be a tame cell in $E$ contained in $B_{1 / 2^{\circ}}$ Then for any $0<\epsilon \leq 1 / 2$, there exists a bomeomorphism $b$ from $E$ onto itself such that $b\left(B_{1 / 2}\right)=$ $C$ and $B_{1-\epsilon} \subset b\left(B_{1-\epsilon}\right) \subset B_{1} \subset b\left(B_{1}\right) \subset B_{1+\epsilon}$.

2. Cellular sets and point-like sets. If $A$ is a subset of $E$, a cellular sequence for $A$ is a decreasing sequence, $\left\{C_{i}\right\}_{i=1}^{\infty}$, of cells in $E$ such that $\bigcap_{i=1}^{\infty} C_{i}=A$ and $C_{i+1} \subset$ Int $C_{i}$ for each $i$. Also $A$ is cellular in $E$ if there exists a cellular sequence for $A$.

If $C$ and $C^{\prime}$ are two closed subsets of $E$ such that $C^{\prime} C$ Int $C$, then $C$ and $C^{\prime}$ are said to have annular difference if there exists a homeomorphism $b$ from the triple $\left(B_{1} \backslash\right.$ Int $\left.B_{1 / 2} ; S_{1}, S_{1 / 2}\right)$ onto the triple $\left(C \backslash\right.$ Int $\left.C^{\prime} ; \mathrm{Bd} C, \mathrm{Bd} C^{\prime}\right)$.

Theorem 2.1. If $A$ is cellular in $E$, then there exists a cellular sequence for $A$ such that each element of the sequence is tame and each two elements of the sequence bave annular difference.

Proof. Let $\left\{C_{i}^{\prime}\right\}_{i=1}^{\infty}$ be a cellular sequence for $A$. For each $i$, by Lemma 1.4, define $C_{i}$ to be a cell in $E$ such that $C_{i} \subset$ Int $C^{\prime}, C_{i+1}^{\prime} \subset$ Int $C_{i}$, and $C_{i}^{\prime} \backslash$ Int $C_{i}$ is a collar of $C_{i}$. Then $\left\{C_{i}\right\}_{i=1}^{\infty}$ is a cellular sequence for $A$ such that each $C_{i} \backslash$ Int $C_{i+1}$ contains a collar of $C_{i+1}$. By Lemma 1.2 , for each $i$, there exists a homeomorphism $b_{i}$ from the pair $\left(B_{1}, S_{1}\right)$ onto the pair $\left(C_{i}, \mathrm{Bd} C_{i}\right)$ such that $C_{i+1} \subset b_{i}\left(B_{1 / 2}\right)$. If $E$ is infinite-dimensional, choose $\sigma>0$ and $w \in E$ such that $B_{\sigma}(w) \subset E \backslash C_{1}$, and let $A_{1}=E \backslash$ Int $B_{\sigma}(w)$. If $E$ is finite-dimensional, let $A_{1}$ be some closed ball containing $C_{1}$. In either case there exists a homeomorphism $f_{1}$ from $E$ onto itself such that $f_{1}\left(B_{1}\right)=A_{1}$.

Suppose that subsets $A_{i}$ of $E$ and homeomorphisms $f_{i}$ from $E$ onto itself have been defined for $1 \leq i \leq k$ so that $f_{i}\left(B_{1}\right)=A_{i}, f_{i}\left(B_{2}\right)=A_{i-1}$, and $C_{i} C$ Int $A_{i} \subset$ Int $C_{i-1}$ (where $A_{0}=f_{1}\left(B_{2}\right)$ and $\left.C_{0}=E\right)$. Then inductively define $A_{k+1}$ and $f_{k+1}$ as follows. Let $\epsilon>0$ and $x \in E$ be chosen so that $B \epsilon(x) \subset f_{k}^{-1}\left(C_{k+1}\right)$. Also choose $\delta>0$ and $y \in E$ so that $B_{\delta}(y) \subset b_{k}^{-1} f_{k}\left(B_{\epsilon}(x)\right)$. Define the homeomorphism $g$ from $B_{1}$ onto itself so that $g\left(B_{\delta}(y)\right)=B_{1 / 2}$ and $\left.g\right|_{S_{1}}=$ identity. Define $G$, a homeomorphism from $E$ onto itself, by $G(x)=f_{k}^{-1} b_{k} g b_{k}^{-1} f_{k}(x)$ if $x \in f_{k}^{-1}\left(C_{k}\right)$, and $G(x)=x$ otherwise. Let $\phi$ be a homeomorphism from $E$ onto itself such that $\phi\left(B_{1}\right)=B_{\epsilon}(x)$ and $\phi\left(B_{2}\right)=B_{1}$. Then set $f_{k+1}=f_{k} G \phi$ and $A_{k+1}$ $=f_{k+1}\left(B_{1}\right)$. Then $\left\{A_{i}\right\}_{i=1}^{\infty}$ is a cellular sequence for $A$ satisfying the conclusion of the theorem. 
A subset of a homogeneous space $X$ is point-like in $X$ if its complement in $X$ is homeomorphic to the complement of a point in $X$. When $E$ is infinite-dimensional, the complement of a point in $E$ is homeomorphic to $E$ [14]. Therefore the property of being point-like in $E$ is equivalent to the property of being negligible in $E$ for infinite-dimensional $E$ (a set is negligible in a space $X$ if its complement is homeomorphic to $X$ ).

It is known that cellular sets are equivalent to connected, point-like sets in finite-dimensional spaces [16]. This is not true for infinite-dimensional spaces, as the following example shows. Let $s$ be the countable infinite product of open intervals. Anders on showed that $s$ is homeomorphic to separable Hilbert space (see [3]). For each $i$, let $K_{i}=\left\{\left(x_{1}, x_{2}, \ldots\right) \in s: x_{j} \in[-1 / 2,1 / 2]\right.$ for $j \leq i$, and $x_{j}=0$ for $\left.j>i\right\}$. Then $K=\bigcup_{i=1}^{\infty} K_{i}$ is a connected $\sigma$-compact subset of $s$ which is not closed in $s$, and hence not cellular in $s$ (Hilbert space). Since $K$ is $\sigma$-compact, by results in [3], $K$ is point-like.

Theorem 2.2. If $E$ is infinite-dimensional, then a subset of $E$ is cellular in $E$ if and only if it is closed and point-like in $E$.

Proof. By Theorem 2.1, if $A$ is cellular in $E$ there exists a cellular sequence $\left\{C_{i}\right\}_{i=1}^{\infty}$ for $A$ such that each $C_{i}$ is tame and each $C_{i}$ and $C_{i+1}$ have annular difference. Since $C_{1}$ is tame, let $b_{1}$ be a homeomorphism from $E$ onto itse lf so that $b_{1}\left(B_{1}\right)=C_{1}$. Since $C_{1}$ and $C_{2}$ have annular difference, there is a homeomorphism $f$ from the triple $\left(B_{1} \backslash\right.$ Int $\left.B_{1 / 2} ; S_{1}, S_{1 / 2}\right)$ onto the triple $\left(C_{1} \backslash\right.$ Int $C_{2} ;$ Bd $C_{1}$, Bd $\left.C_{2}\right)$. Define a homeomorphism $g$ from the pair $\left(B_{1} \backslash\right.$ Int $\left.B_{1 / 2}, S_{1 / 2}\right)$ onto itself so that $\left.g\right|_{S_{1}}=\left.f^{-1} b_{1}\right|_{s_{1}}$. Set $b_{2}=f$, which is a homeomorphism from the triple $\left(B_{1} \backslash\right.$ Int $\left.B_{1 / 2} ; S_{1}, S_{1 / 2}\right)$ onto the triple $\left(C_{1} \backslash\right.$ Int $\left.C_{2} ; B d C_{1}, B d C_{2}\right)$ such that $\left.b_{2}\right|_{s_{1}}$ $=\left.b_{1}\right|_{s_{1}}$. Then by induction, define, for each $n>1$, the homeomorphism $b_{n}$ from the triple $\left(B_{1 /(n-1)} \backslash\right.$ Int $\left.B_{1 / n} ; S_{1 /(n-1)}, S_{1 / n}\right)$ onto the triple $\left(C_{n-1} \backslash\right.$ Int $C_{n}$; Bd $C_{n-1}$, Bd $\left.C_{n}\right)$ such that $\left.b_{n}\right|_{S_{1 /(n-1)}}=\left.b_{n-1}\right|_{S_{1 /(n-1)}}$. The desired homeomorphism $b$ from $E \backslash\{\theta\}$ onto $E \backslash A$ may now be defined by $b(x)=b_{1}(x)$ if $x \in$ $E \backslash B_{1}$, and $h(x)=b_{n}(x)$ if $x \in B_{1 /(n-1)} \backslash B_{1 / n}$ for $n>1$.

Conversely, let $A$ be a closed and point-like in $E$. Since $E$ is infinitedimensional, there exists a homeomorphism $f$ from $E$ onto $E \backslash A$. For each positive integer $i$, define $V_{i}=\{x \in E \backslash A: d(x, A) \leq 1 / i\}$. Then $\left\{V_{i}\right\}_{i=1}^{\infty}$ is a decreasing sequence of closed sets in $E \backslash A$, and since $A$ is closed, $\bigcap_{i=1}^{\infty} V_{i}=\varnothing$. It can be assumed without loss of generality that $f^{-1}\left(V_{1}\right) \cap B_{1}=\varnothing$. For each $i$, let $N_{i}=\left\{x \in E: d\left(x, f^{-1}\left(V_{i}\right)\right)<1 / i\right\}$. By Urysohn's lemma, there exists a continuous function $r_{i}: E \rightarrow[0,1]$ such that $r_{i}\left(f^{-1}\left(V_{i}\right)\right)=1$ and $r_{i}\left(E \backslash N_{i}\right)=0$. Define the continuous function $r: E \rightarrow[1, \infty)$ by $r(x)=\max \left\{1, \sum_{i=1}^{\infty} r_{i}(x)\right\}$. It can be seen that $r\left(f^{-1}\left(V_{i}\right)\right) \subset[i, \infty)$ for each $i$. Define the homeomorphism $g$ from $E$ 
onto itse lf by $g(x)=s x$, where $s=\sup \{t: t \in r([\theta: x])\}$, so that $B_{i} \cap g f^{-1}\left(V_{i}\right)=$ $\varnothing$. Then for each $i$, define $C_{i}=f g^{-1}\left(B_{i}\right)$. Since $C_{i} \cap V_{i}=\varnothing$, each $C_{i}$ is closed and is hence a cell. Also $f\left(B_{i+1} \backslash\right.$ Int $\left.B_{i}\right)$ is a collar of $C_{i}$ in $E$ for each $i$. Therefore by Theorem 1.1, each $C_{i}$ is tame in $E$. By the result of Klee in [14], there is a homeomorphism $\phi$ from $E$ onto itself so that $\phi\left(B_{1}\right)=E \backslash$ Int $B_{1}$. Thus, if $A_{i}=E \backslash$ Int $C_{i}$, each $A_{i}$ is a cell in $E$. Since $\bigcap_{i=1}^{\infty} A_{i}=A, A$ is cellular in $E$.

The following kinds of sets have been shown to be negligible, and hence point-like. Klee showed in [14] that compact subsets of infinite-dimensional $E$ are negligible. Anderson, Henderson, and West showed in [4] that closed subsets of separable Hilbert space which have Property $Z$ (equivalently, topological infinite deficiency) are negligible. Also Cutler showed in [11] that countable unions of locally closed, locally infinite-deficient subsets of a complete space $E$ are negligible, and countable unions of locally compact subsets of nonseparable Hilbert spaces are negligible.

3. Strongly cellular sets and decomposition spaces. A subset $A$ of $E$ is strongly cellular in $E$ if there exists a cellular sequence, $\left\{C_{i}\right\}_{i=1}^{\infty}$, for $A$ such that for each open set $U$ in $E$ containing $A$, there exists an integer $n$ such that $C_{n} \subset U$. Such a cellular sequence will be called a strongly cellular sequence for A.

In finite-dimensional spaces, cellularity and strong cellularity are equivalent and agree with the usual definition of cellularity there. However, in general they are not equivalent. In fact, it will be seen that strongly cellular sets must be compact and connected. The following is an example of a cellular set which is neither compact nor connected, and hence not strongly cellular. Let $E$ be infinitedimensional and let $x, y \in E$. Define $A$ to be the line segment from $x$ to $y$, which is cellular in $E$; in fact any compact convex subset of $E$ is strongly cellular in $E$. Let $\left\{C_{i}\right\}_{i=1}^{\infty}$ be a cellular sequence for $A$, with $b_{i}$ a homeomorphism from the pair $\left(B_{1}, S_{1}\right)$ onto the pair $\left(C_{i}, B d C_{i}\right)$ for each $i$. Also for each $i$, let $g_{i}$ be a homeomorphism from $B_{1}$ onto $B_{1} \backslash\left\{h_{i}^{-1}(z)\right\}$ (where $z=1 / 2 x+1 / 2 y$ ) such that $\left.g_{i}\right|_{s_{1}}=$ identity. Define $f$ to be some homeomorphism from $E \backslash\{z\}$ onto $E$, and set $C=$ $f(A \backslash\{z\})$. Then $C$ is not compact or connected, but $\left\{f b_{i} g_{i}\left(B_{1}\right)\right\}_{i=1}^{\infty}$ is a cellular sequence for $C$.

Let $D$ be a decomposition of $E$ into compact sets, and let $H[D]$ be the class of nondegenerate elements of $D$. $E / D$ denotes the decomposition space of $E$ defined by $D$. If $H[D]$ consists of the single element $A$, then $E / A$ will be used to denote $E / D$. $D$ will be called strongly cellular if every element of it is strongly cellular in $E$.

When $E$ is finite-dimensional, it is known that $A$ is cellular in $E$ if and only 
if $E / A$ is homeomorphic to $E[16]$. There is a corresponding relationship for strongly cellular sets in infinite-dimensional normed linear spaces.

Theorem 3.1. Let $D$ be a decomposition of $E$ such that $H[D]$ is discrete. Then $E / D$ is homeomorphic to $E$ if and only if $D$ is strongly cellular.

Proof. Let $D=\left\{D_{\gamma} \mid \gamma \in \Gamma\right\}$. Since $E$ is collectionwise normal there exists a pairwise disjoint collection $\mathcal{U}=\left\{U_{\gamma} \mid \gamma \in \Gamma\right\}$ of open subsets of $E$ such that $D_{\gamma} \subset U_{\gamma}$ for each $\gamma \in \Gamma$. First let $f$ be a homeomorphism from $E / D$ onto $E$, let $g$ be the canonical map from $E$ onto $E / D$, let $b=f g$, let $A \in H[D]$, and let $V$ be the member of $\mathcal{U}$ containing $A$. Without loss of generality assume that $b(A)=$ $\{\theta\}$. Choose $\epsilon>0$ so that $B_{\epsilon} \subset b(U)$, and for each $i$, define $A_{i}=b^{-1}\left(B_{\epsilon / i}\right)$. If $E$ is infinite-dimensional, $E \backslash A_{i}$ is a cell in $E$ for each $i$ (the finite-dimensional case can be established by appealing to the Schoenflies theorem-Sanderson has proved an infinite-dimensional Schoenflies Theorem in [15] which could be used here in the infinite-dimensional case also). Therefore, since $E \backslash A$ is open, each $b^{-1}\left(B_{\epsilon / i} \backslash\right.$ Int $\left.B_{\epsilon /(i+1)}\right)$ is a collar of $E \backslash \Lambda_{i}$ in $E \backslash \Lambda$. Thus each $E \backslash B_{i}$ is tame, so that $A_{i}$ is a cell in $E$. Since $\bigcap_{i=1}^{\infty} A_{i}=\Lambda,\left\{\Lambda_{i}\right\}_{i=1}^{\infty}$ is a strongly cellular sequence for $A$.

Conversely, let $\gamma_{0}$ be a fixed element of $\Gamma$, and let $\Lambda=D_{\gamma_{0}}$ and $V=U_{\gamma_{0}}$. By the proof of Theorem 2.1, there exists a strongly cellular sequence $\left\{\Lambda_{i}\right\}_{i=1}^{\infty}$ for $\Lambda$ which is contained in $V$ and such that each $\Lambda_{i}$ is tame and each two of its elements have annular difference. Let $g$ be a homeomorphism from the triple $\left(B_{1} ; S_{1}, S_{1 / 2}\right)$ onto the triple $\left(\Lambda_{1} ; \mathrm{Bd} \Lambda_{1}, \mathrm{Bd} \Lambda_{2}\right)$. Call $x_{\gamma_{0}}=g(\theta)$, and set $C_{i}=$ $g\left(B_{1 / i}\right)$ for each $i$ (note that $C_{1}-\Lambda_{1}$ and $C_{2}-\Lambda_{2}$ ). Let $b_{0}$ be the identity map from $E \backslash$ Int $\Lambda_{1}$ onto itself and $b_{1}$ the identity from $\Lambda_{1} \backslash$ Int $\Lambda_{2}$ onto itself. Suppose that, for $1 \leq i \leq k-1$, homeomorphisms $b_{i}$ have been defined from $\Lambda_{i} \backslash$ Int $A_{i+1}$ onto $C_{i} \backslash$ Int $C_{i+1}$ such that $\left.b_{i}\right|_{\mathrm{Bd} A_{i}}=\left.b_{i-1}\right|_{\mathrm{Bd} A_{i}}$. Then define $b_{k}$ from $A_{k} \backslash$ Int $\Lambda_{k+1}$ onto $C_{k} \backslash$ Int $C_{k+1}$ such that $\left.b_{k}\right|_{\mathrm{Bd} A_{k}}=\left.b_{k-1}\right|_{\mathrm{Bd} A_{k}}$ as follows. Since $\Lambda_{k}$ and $\Lambda_{k+1}$ have annular difference, there exists a homeomorphism $f$ from the triple $\left(B_{1 / k} \backslash\right.$ Int $\left.B_{1 /(k+1)} ; s_{1 / k}, s_{1 /(k+1)}\right)$ onto the triple $\left(\Lambda_{k} \backslash\right.$ Int $\Lambda_{k+1}$; Bd $\left.\Lambda_{k}, \mathrm{Bd} \Lambda_{k+1}\right)$. For $x \in \Lambda_{k} \backslash$ Int $\Lambda_{k+1}$ define $b_{k}(x)=g\left(\left[\left\|f^{-1}(x)\right\| /\|y\|\right] y\right)$, where $y=g^{-1} b_{k-1} f\left(\left[1 / k\left\|f^{-1}(x)\right\|\right] f^{-1}(x)\right)$. Then with the $\left\{b_{i}\right\}_{i=1}^{\infty}$ inductively defined, define the homeomorphism $b_{y_{0}}$ from $E \backslash \Lambda$ onto $E \backslash\left\{x_{\gamma_{0}}\right\}$ by $b_{\gamma_{0}}(x)=b_{0}(x)$ if $x \in E \backslash$ Int $\Lambda_{1}$, and $h_{\gamma_{0}}(x) \cdot h_{i}(x)$ if $x \in \Lambda_{i} \backslash$ Int $\Lambda_{i+1}$.

Thus for each $\gamma \in \mathrm{I}$, a homcomorphism $b_{\gamma}$ is defined from $E \backslash D_{\gamma}$ onto $E \backslash\left\{x_{y}\right\}$ which is the identity on the boundary of ${ }^{\prime} \gamma$, where $x_{y} \in \|_{\gamma}$. Define the desired homeomorphism $b$ from $E / D$ onto $I:$ by $h(x)-x_{y}$ if $x=D y, b(x)$ $b_{\gamma}(x)$ if $x \in U_{\gamma} \backslash D_{\gamma}$, and $b(x)=x$ if $x \in E \backslash \bigcup\left\{U_{\gamma}: \gamma \in \Gamma\right\}$. The strong cellularity is necessary in order that $h^{-1}$ be continuous at each $x_{\gamma}$. 
It has been asked whether the decomposition space defined from any compact upper semicontinuous decomposition of Hilbert space is homeomorphic to Hilbert space. More generally, consider $E$ any normed linear space. Let $\Sigma$ be a subset of $E$ which is the homeomorphic image of an $n$-sphere. It will follow from Corollary 3.1 that $\Sigma$ is not strongly cellular in $E$. Thus, by Theorem $3.1, E / \Sigma$ is an example of a decomposition space defined from a compact upper semicontinuous decomposition of $E$ which is not homeomorphic to $E$.

The concept of "shape of a compactum" used in the following theorem can be found for example in [7].

Theorem 3.2. Every strongly cellular set in $E$ is a compactum with the shape of a point. Conversely, if $E$ is homeomorphic to the product of itself with Hilbert space, then every compactum with the shape of a point is strongly cellular. (2)

Proof. Let $A$ be strongly cellular in $E$ and let $\left\{C_{i}\right\}_{i=1}^{\infty}$ be a strongly cellular sequence for $A$. It is clear that $A$ is compact if $E$ is finite-dimensional, so let $E$ be infinite-dimensional. Now suppose that Int $A \neq \varnothing$. Since $E \backslash A$ is nonempty and open, there is a homeomorphism from $E$ onto itself taking $\Lambda$ into some ball contained in $E \backslash A$ (due to results in [14]). Hence it can be assumed without loss of generality that $A$ is bounded in $E$ and $\theta \in \operatorname{Int} A$. Let $\left\{x_{i}\right\}_{i=1}^{\infty}$ be a sequence of points of $S_{1}$ in $E$ which has no limit point. For each integer $i$, there exists $y_{i} \in\left(C_{i} \cap T_{i}\right) \backslash A$, where $T_{i}$ is the half-infinite ray starting at $\theta$ and passing through $x_{i}$. Then $E \backslash\left\{y_{i}\right\}_{i=1}^{\infty}$ is open, contains $A$, and contains no $C_{i}$. This is a contradiction, so that Int $A=\varnothing$. Next suppose that $A$ is not compact. Let $\left\{z_{i}\right\}_{i=1}^{\infty}$ be a sequence of points of $A$ which has no limit point, and let $\left\{U_{i}\right\}_{i=1}^{\infty}$ be a mutually disjoint sequence of open subsets of $E$ such that $z_{i} \in U_{i}$ and $U_{i} \subset B_{1 / 2 i}\left(z_{i}\right)$ for each $i_{\text {o Since }} U_{i}$ is not contained in $A$ because Int $A=\varnothing$, for each integer $i$ there exists a $w_{i} \in\left(C_{i} \cap U_{i}\right) \backslash A$. Then $E \backslash\left\{w_{i}\right\}_{i=1}^{\infty}$ is open, contains $A$, and contains no $C_{i}$. This is again a contradiction, so that $A$ is compact. Since each neighborhood of $A$ contains a cell containing $A, A$ is contractible in every neighborhood. Then by Corollary 9.5 in [6], $A$ is a fundamental absolute retract. Therefore by Theorem 7.1 in [7], $A$ has the shape of a point.

Conversely, suppose that $E$ is homeomorphic to the product of itself with Hilbert space, and $A$ is a compactum with the shape of a point. Hilbert space is homeomorphic to $s$, the countable infinite product of lines, [3], and $s$ is homeomorphic to $s \times Q[1]$, where $Q$ is the Hilbert cube. Thus there exists a

(2) The author would like to thank the referee for pointing out Theorem 3.2 , which amalgamates and strengthens several of the author's original theorems. 
homeomorphism $\phi$ from $E$ onto $E \times Q$. Since $A$ is compact, it may be assumed that $\phi(A) \subset\{\theta\} \times W \subset E \times Q$, where $W$ has infinite deficiency in $Q$ (see [2]). By a theorem of Chapman [9], considering $\{\theta\} \times Q$ as $Q$, there exists a pseudoisotopy $H:(\{\theta\} \times Q) \times I \rightarrow(\{\theta\} \times Q) \times I$, where $H$ is a level-preserving surjection which is a homeomorphism on the complement of $\phi(A) \times\{0\}$ and which maps $\phi(A)$ $\times\{0\}$ to a point. For $q \in Q$, let $\bar{q}$ denote $(\theta, q) \in\{\theta\} \times Q$. Let $H^{*}(\bar{q}, t)$ be that element of $Q$ such that $H(\bar{q}, t)=\left(\left(\theta, H^{*}(\bar{q}, t)\right), t\right)$. Define the homeomorphism $b$ from $(E \times Q) / \phi(A)$ onto $E \times Q$ as follows. Let $(x, q) \in(E \times Q) / \phi(A)$. If $(x, q) \neq$ $\phi(A)$ and $0 \leq\|x\| \leq 1$, define $b(x, q)=\left(x, H^{*}(\bar{q},\|x\|)\right)$. If $(x, q)=\phi(A)$, define $b(x, q)=\left(\theta, H^{*}(a, 0)\right)$, where $a \in A$. Finally, if $\|x\|>1$, define $b(x, q)=(x, q)$. With $b$ thus defined, $\phi^{-1} b \phi^{\prime}$ is a homeomorphism from $E / A$ onto $E$, where $\phi^{\prime}$ is the homeomorphism from $E / A$ onto $(E \times Q) / \phi(A)$ induced by $\phi$. Therefore by Theorem 3.1, $A$ is strongly cellular in $E$.

The condition that $E$ is homeomorphic to the product of itself with Hilbert space cannot be deleted since a wild arc in $R^{3}$ has the shape of a point, but is not cellular in $R^{3}$.

The following corollary of Theorem 3.2 is a consequence of results in [5].

Corollary 3.1. Let $A$ be a strongly cellular subset of $E$. Then $A$ bas the bomology groups of a point. Further, if $A$ is an absolute neighborbood retract, then it bas the bomotopy groups of a point.

Corollary 3.2. If $E$ is homeomorphic to the product of itself and Hilbert space, then every subset of $E$ which is bomeomorphic to some compact point-like subset of $R^{n}$ is strongly cellular in $E$.

4. The monotone union of open cells. The purpose of this section is to obtain an analog to Brown's theorem which says that the union of an increasing sequence of open $n$-cells is an $n$-cell [8].

An open $E$-cell in a topological space $X$ is defined to be an open subset of $X$ which is homeomorphic to $E$. If a subset $Q$ of $E$ is an open $E$-cell in $E$, then $Q$ will be said to be an open cell in $E$. The space $E$ has the monotone union property provided the following is true. If $\left\{Q_{i}\right\}_{i=1}^{\infty}$ is an increasing sequence of open $E$-cells in any space $X$, then $\bigcup_{i=1}^{\infty} Q_{i}$ is an open $E$-cell in $X$.

Lemma 4.1. If $\left\{Q_{i}\right\}_{i=1}^{\infty}$ is an increasing sequence of open E-cells in $X$, then $\bigcup_{i=1}^{\infty} Q_{i}$ bas trivial bomotopy type.

Proof. Let $n$ be an arbitrary nonnegative integer, and let $f$ be a map from the standard $n$-sphere $S^{n}$ into $\bigcup_{i=1}^{\infty} Q_{i}$. Since $f\left(S^{n}\right)$ is compact, $f\left(S^{n}\right) \subset Q_{m}$ for some integer $m$. Since $Q_{m}$ is an open $E$-cell, $f$ extends to a map from the standard $(n+1)$-ball $B^{n+1}$ into $Q_{m}$. 
Theorem 4.1. If $E$ is homeomorphic to the countable infinite product of copies of itself, then $E$ bas the monotone union property.

Proof. Let $\left\{Q_{i}\right\}_{i=1}^{\infty}$ be an increasing sequence of open $E$-cells in $X$. Then $\bigcup_{i=1}^{\infty} Q_{i}$ is an $E$-manifold, and by Lemma 4.1 , it has the homotopy type the same as that of $E$. It has been established that two $E$-manifolds are homeomorphic if and only if they have the same homotopy type (see [13]). Therefore $\bigcup_{i=1}^{\infty} Q_{i}$ is homeomorphic to $E$, and hence is an open $E$-cell in $X$.

\section{REFERENCES}

1. R. D. Anderson, Topological properties of the Hilbert cube and the infinite product of open intervals, Trans. Amer. Math. Soc. 126 (1967), 200-216. MR 34 \#5045.

2. - On topological infinite deficiency, Michigan Math. J. 14 (1967), 365-383. MR $35 \# 4893$.

3. R. D. Anderson and R. H. Bing, A complete elementary proof that Hilbert space is homeomorphic to the countable infinite product of lines, Bull. Amer. Math. Soc. 74 (1968), 771-792. MR 37 \#5847.

4. R. D. Anderson, D. W. Henderson and J. E. West, Negligible subsets of infinitedimensional manifolds, Compositio Math. 21 (1969), 143-150. MR 39 \#7630.

5. K. Borsuk, Concerning homotopy properties of compacta, Fund. Math. 62 (1968), 223-254. MR $37 \# 4811$.

6. - Fundamental retracts and extensions of fundamental sequences, Fund. Math. 64 (1969), 55-85; Errata, ibid. 375. MR 39 \#4841.

7. - A note on the theory of shape of compacta, Fund. Math. 67 (1970), 265278. MR $42 \# 1077$.

8. M. Brown, The monotone union of open n-cells is an open n-cell, Proc. Amer. Math. Soc. 12 (1961), 812-814. MR 23 \#A4129.

9. T. Chapman, On some applications of infinite-dimensional manifolds to the theory of shape (to appear).

10. D. W. Curtis and R. A. McCoy, Stable homeomorphisms on infinite-dimensional normed linear spaces, Proc. Amer. Math. Soc. 28 (1971), 496-500.

11. W. H. Cutler, Negligible subsets of infinite-dimensional Fréchet manifolds, Proc. Amer. Math. Soc. 23 (1969), 668-675. MR 40 \#2133.

12. C. Greathouse, Locally flat strings, Bull. Amer. Math. Soc. 70 (1964), 415-418. MR $28 \# 4526$.

13. D. W. Henderson and R. Schori, Topological classification of infinite-dimensional manifolds by homotopy type, Bull. Amer. Math. Soc. 76 (1970), 121-124. MR 40 \#4976.

14. V. L. Klee, A note on topological properties of normed linear spaces, Proc. Amer. Math. Soc. 7 (1956), 673-674. MR 17, 1227.

15. D. E. Sanderson, An infinite-dimensional Schoenflies theorem, Trans. Amer. Math. Soc. 148 (1970), 33-39. MR 41 \#4586.

16. University of Georgia, Summer Institute in Topology 1961 (notes).

DEPARTMENT OF MATHEMATICS, IOWA STATE UNIVERSITY OF SCIENCE AND TECHNOLOGY, AMES, IOWA 50010

DEPARTMENT OF MATHEMATICS, VIRGINIA POLYTECHNIC INSTITUTE AND STATE UNIVERSITY, BLACKSBURG, VIRGINIA 24061 